\title{
The Chk1 inhibitor AZD7762 sensitises p53 mutant breast cancer cells to radiation in vitro and in vivo
}

\author{
ZHIKUN MA ${ }^{1}$, GUOLIANG YAO ${ }^{2}$, BO ZHOU ${ }^{1}$, YONGGANG FAN ${ }^{2}$, \\ SHEGAN GAO ${ }^{1}$ and XIAOSHAN FENG ${ }^{1}$
}

\author{
Departments of ${ }^{1}$ Oncology and ${ }^{2}$ General Surgery, The First Affiliated Hospital of the Henan \\ University of Science and Technology, Luoyang, Henan 471003, P.R. China
}

Received April 10, 2012; Accepted July 16, 2012

DOI: $10.3892 / \mathrm{mmr} .2012 .999$

\begin{abstract}
AZD7762, a novel checkpoint kinase 1 (Chk 1) inhibitor, has been proven to sensitize various tumor cells to DNA damage. However, whether or not AZD7762 sensitizes breast cancer cells to radiation has not been defined. In the present study, we aimed to demonstrate for the first time, that AZD7762 not only promotes radiation-induced apoptosis and mitotic catastrophe of p53 mutant T47D breast cancer cells in vitro, but also delays their xenograft growth in response to radiation in vivo. Our mechanistic study showed that AZD7762 treatment resulted in the abrogation of radiation-induced G2/M arrest and the inhibition of radiation damage repair as demonstrated by increased radiation-induced $\gamma \mathrm{H} 2 \mathrm{AX}$ expression and decreased RAD51 protein expression. These results suggest that AZD7762 may effectively abrogate radiation-induced $\mathrm{G} 2 / \mathrm{M}$ arrest and inhibit radiation damage repair in conferring radiosensitivity on p53 mutant T47D breast cancer cells, by promoting radiation-induced apoptosis and mitotic catastrophe. The clinical application of AZD7762, as an adjuvant in the radiotherapy of breast cancers, should be further explored.
\end{abstract}

\section{Introduction}

Radiotherapy has been adopted as the standard treatment of breast cancer (1). However, its overall efficiency is unsatisfactory due to radiation resistance (2). When tumor cells suffer radiation-induced DNA damage, the DNA damage checkpoints are activated and once activated may lead to cell cycle arrest, allowing the repair of the DNA damage, which in turn promotes the development of radiation resistance (3-5).

In the activity of DNA damage checkpoints, checkpoint kinase 1 (Chk1) has been described as a central mediator. The activation of Chk1, not only phosphorylates the CDC25

Correspondence to: Dr Xiaoshan Feng, Department of Oncology, The First Affiliated Hospital of the Henan University of Science and Technology, 24 Jinghua Rd, Luoyang, Henan 471003, P.R. China E-mail: oncoly@yahoo.cn

Key words: AZD7762, breast cancer, checkpoint kinase 1, p53 mutant, apoptosis family leading to $\mathrm{G} 2 / \mathrm{M}$ phase arrest, but also promotes Rad51-mediated DNA double strand break repair (6). Thus, Chk1 is crucial in the development of radiation resistance, and as a result, the inhibition of Chk1 may lead to a preferential radiation-induced killing of tumor cells. Experimental data in the literature also suggest that this inhibition applies to numerous tumor cells (7-10). As a new model of Chk1 inhibitor, AZD7762 has been demonstrated to sensitize many tumor cell lines in anticancer drug therapy (11-13). However, whether the Chk1 inhibitor promotes radiation-induced tumor cell death in breast cancer cells remains to be determined.

The aim of this study was to determine whether the Chk1 inhibitor AZD7762 is capable of enhancing the toxicity of radiation to cells in vitro and on xenografts in vivo using p53 mutant T47D cells and wild-type p53 MCF7 cells of human breast cancer. Results showed that AZD7762 treatment significantly potentiated the lethal effects of radiation on p53 mutant T47D cells, but not on wild-type p53 MCF7 cells in vitro and in vivo. Thus, the Chk1 inhibitor AZD7762 has the potential to improve the efficacy of radiation therapy in human breast cancer with p53 mutations.

\section{Materials and methods}

Cell lines and nude mice. The p53 mutant T47D and wild-type p53 MCF7 cell lines were used in this study. The two cell lines were obtained from the cell culture center of the Wuhan University and were kept in DMEM/F12 culture medium supplemented with $10 \%$ fetal bovine serum (FBS), penicillin $(100 \mu \mathrm{g} / \mathrm{ml})$ and streptomycin $(100 \mu \mathrm{g} / \mathrm{ml})$ in a humidified incubator $\left(37^{\circ} \mathrm{C}, 5 \%\right.$ carbon dioxide). Animal experiments were approved by the Local Animal Care and Use Committee and were conducted in accordance with the NIH guidelines. As the treatment of AZD7762 (100 nmol/1 in vitro and $25 \mathrm{mg} /$ $\mathrm{kg}$ in vivo) has been reported to yield maximal radiation enhancement with minimal cytotoxicity to other tumor cells in previous studies, in the present study, AZD7762 was used in vitro and in vivo at concentrations of $100 \mathrm{nmol} / 1$ and 25 $\mathrm{mg} / \mathrm{kg}$, respectively (14). Nude mice, 4- to 6-weeks old, were inoculated subcutaneously on the hind flank with tumor cells $\left(1 \times 10^{5}\right)$. One week later, xenografts were formed. Subsequently, the mice were randomized into 4 groups with 4 mice in each group. The control group received PBS i.p. treatment twice 
a day for 4 days. The AZD7762 group received AZD7762 treatment $(25 \mathrm{mg} / \mathrm{kg}$, i.p., AstraZeneca) twice a day for 4 days only. In the radiation group and the radiation plus AZD7762 group, mice were radiated once a day for 4 days with a dose of 2 Gy per fraction (total dose: $8 \mathrm{~Gy}$ ). During the radiation treatment, mice in the radiation plus AZD7762 group were injected with AZD7762 (25 mg/kg, i.p., AstraZeneca) twice a day for 4 days. Tumor size was assessed by caliper measurements twice a week. Volumes were calculated using the formula $(\mathrm{LxWxW}) / 2$. Three weeks after radiation, the mice were sacrificed and xenografts were excised for analysis.

Cell clonogenic assays. The two cell lines were seeded into 6-well plates at a density of 1000 cells per well in the presence or absence of AZD7762 treatment. Cells were treated with AZD7762 (100 nmol/l) $1 \mathrm{~h}$ prior to, and left on for $24 \mathrm{~h}$ after radiation (8 Gy). Subsequently, AZD7762 was removed and the fresh culture medium was added. Ten days after radiation, the cells were fixed and a clonogenic assay was carried out. Cell culture medium was removed for analysis and cells were washed once, using PBS. The cells were then fixed in $75 \%$ methanol and $25 \%$ acetic acid (vol $/ \mathrm{vol}$ ) for $15 \mathrm{~min}$. Colonies were stained for 20 min with $0.5 \%$ crystal violet in methanol. After washing and drying, the colonies were counted. Clonogenicity was expressed as \% colonies relative to untreated cells. Average values were presented as the mean $\pm \mathrm{SD}$. The clonogenic experiments were performed in triplicate.

Cell cycle analysis. Since the changes of the cell cycle in many tumor cells become apparent $12 \mathrm{~h}$ after DNA damage, T47D cells were treated with AZD7762 $(100 \mathrm{nmol} / \mathrm{l}) 1 \mathrm{~h}$ prior to, and left on for $12 \mathrm{~h}$ after radiation ( $8 \mathrm{~Gy}$ ) (15). The cells were then collected and centrifuged at $1000 \mathrm{rpm}$ for $5 \mathrm{~min}$ and were fixed in $75 \%$ ethanol at $4^{\circ} \mathrm{C}$ overnight. The following day, after centrifugation (1000 rpm for $5 \mathrm{~min}$ ), the cells were suspended using $20 \mu 1$ PBS. After adding $10 \mu 1$ RNase A $(5 \mathrm{mg} / \mathrm{ml})$, the cells were incubated in a $37^{\circ} \mathrm{C}$ water bath for $30 \mathrm{~min}$. Additional cells with PI $(500 \mu \mathrm{g} / \mathrm{ml})$ were added and incubated for $30 \mathrm{~min}$ in the darkness at room temperature. Flow cytometry was employed for cell cycle analysis. Each experiment was repeated 3 times.

Flow cytometric analysis of apoptosis. T47D cells were treated with AZD7762 (100 nmol/l) $1 \mathrm{~h}$ prior to, and left on for $24 \mathrm{~h}$ after radiation (8 Gy). The cells were then suspended in Annexin V-FITC binding buffer $(195 \mu \mathrm{l})$ and incubated with Annexin V-FITC (5 $\mu \mathrm{l})$ in the dark at room temperature for $10 \mathrm{~min}$. After centrifugation (1000 rpm for $5 \mathrm{~min}$ ), the cells were suspended in binding buffer $(190 \mu \mathrm{l})$ and $10 \mu \mathrm{l}$ PI solution on ice in the darkness. Cell apoptosis was then determined using flow cytometry. The apoptotic index was calculated by the upper and lower right quadrants. Samples were assayed in triplicate.

Assessment of mitotic catastrophe. T47D cells were treated with AZD7762 (100 nmol/l) $1 \mathrm{~h}$ prior to, and left on for $24 \mathrm{~h}$ after radiation ( $8 \mathrm{~Gy}$ ). The medium was removed and the cells were fixed with cold methanol and stained with 4'-6-diamidino-2-phenylindole (DAPI) for chromosome analysis under an Olympus BX41 fluorescence microscope.
Nuclear fragmentation was defined as the presence of $\geq 2$ distinct lobes within a single cell. For each assessment of the extent of mitotic catastrophe, 200 nuclei were examined. Each experiment was repeated 3 times.

Immunohistochemistry analysis. The tumor tissues were collected and fixed in 10\% neutral-buffered formalin, embedded in paraffin and sectioned. Sections were deparaffinized and rehydrated through xylene and a series of alcohols, microwaved in $10 \mathrm{mM}$ citrate buffer $(\mathrm{pH} \mathrm{6.0)}$ for antigen retrieval, endogenous peroxidase was blocked with $3 \%$ hydrogen peroxide and non-specific binding was blocked with $10 \%$ normal goat serum in PBS. Sections were incubated with PCNA antibody (1:2000; Santa Cruz Biotech, Santa Cruz, CA, USA) overnight in a humidity chamber at $4^{\circ} \mathrm{C}$, then incubated again with biotinylated goat anti-mouse $\mathrm{IgG}$, followed by $\mathrm{ABC}$ reagent (Vector Laboratories, Burlingame, CA, USA) and diaminobenzidine (DAB). Sections were lightly counterstained with Harris' hematoxylin and mounted with a glass coverslip for light microscopy.

TUNEL staining. The sections from paraffin-embedded tumors were subjected to TUNEL staining using the In Situ Apoptosis Detection Kit (Millipore, Billerica, MA, USA) according to the manufacturer's instructions. Briefly, sections were deparaffinized and treated with $10 \mu \mathrm{g} / \mathrm{ml}$ proteinase $\mathrm{K}$ in PBS for 10 min, endogenous peroxidase was quenched in 3\% $\mathrm{H}_{2} \mathrm{O}_{2}$ in methanol for $30 \mathrm{~min}$, and the slides were washed in PBS and preincubated in TdT Reaction Buffer for $10 \mathrm{~min}$. The slides were then incubated in TdT Reaction Mixture for $1 \mathrm{~h}$ at $37^{\circ} \mathrm{C}$ in a humid chamber. The reaction was paused in a stop wash buffer for $10 \mathrm{~min}$. Incubated sections were detected with Streptavidin-HRP in PBS for 20 min at room temperature. The color reaction was developed using a DAB substrate. Sections were counterstained with hematoxylin before mounting. Cell numbers were converted into percentage of apoptotic cells calculated from total cell numbers.

Western blot analysis. Tumors or cells were homogenized at $4^{\circ} \mathrm{C}$ in lysis buffer to extract total protein. The protein was quantified using a bicinchoninic acid (BCA) reagent (Thermo Scientific, Rockford, IL, USA). Equal samples (30 $\mu \mathrm{g}$ protein) were electrophoresed through 10-12\% SDS-PAGE gels and were transferred to nitrocellulose paper. The membranes were blocked in 5\% non-fat powdered milk/Tris-buffered saline/Tween-20 (TBST) for $1 \mathrm{~h}$ and incubated overnight with relevant primary antibodies, anti-Chk1 antibody (1:1000, Santa Cruz Biotech), anti-Rad51 antibody (1:1000, Santa Cruz Biotech), anti- $\gamma \mathrm{H} 2 \mathrm{AX}$ antibody (1:500, Upstate, Billerica, MA, USA) and anti- $\beta$-actin antibody (1:3000, Santa Cruz Biotech). After washing, the membranes were incubated in secondary antibody-HRP conjugates for $1.5 \mathrm{~h}$ at room temperature. Blots were then washed and visualized by enhanced chemiluminescence (Thermo Scientific).

Statistical analysis. Statistical analysis was conducted using the SPSS 16.0 software. Continuous variables were expressed as the means $\pm \mathrm{SD}$. The Student's t-test and variance analysis were used in this study. $\mathrm{P}<0.05$ was considered to indicate a statistically significant difference. 

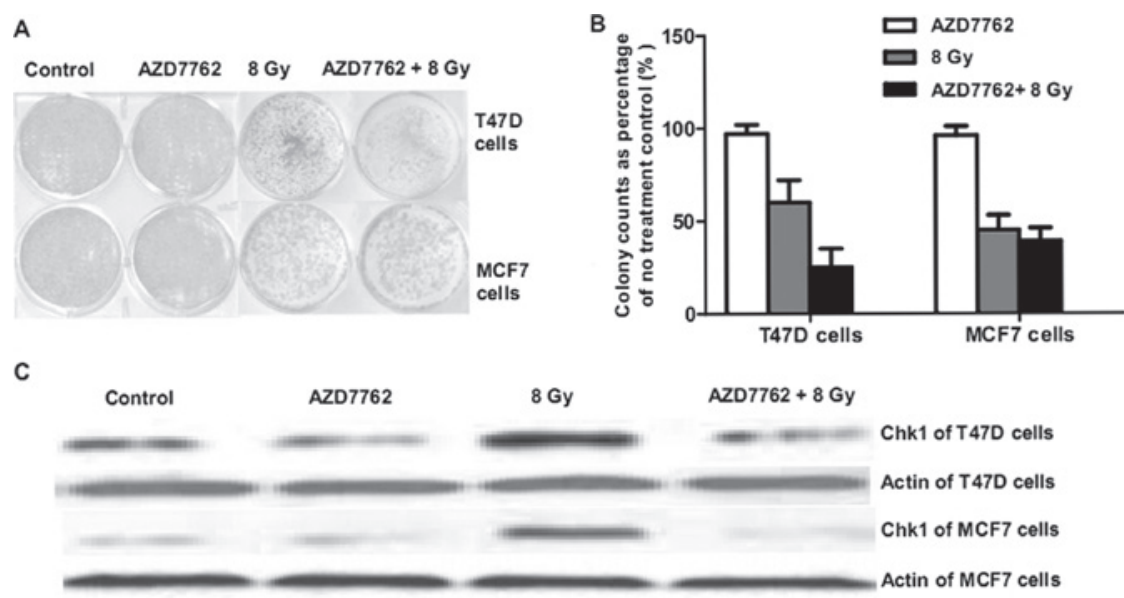

Figure 1. Analysis of clonogenic survival in human breast cancer cells exposed to radiation and/or AZD7762 in vitro. (A) Representative images of clonogenic assays. Cells were seeded into 6-well plates at a density of 1000 cells per well. After attachment, AZD7762 (100 nmol/1) or the vehicle control was added and the plates were irradiated ( $8 \mathrm{~Gy}$ ) after $1 \mathrm{~h}$, while AZD7762 was left on for $24 \mathrm{~h}$ after radiation ( 8 Gy). Ten days after radiation, the cells were fixed and a clonogenic assay was carried out. Clonogenic assays are the average of 3 independent experiments. (B) The bar graph demonstrates that AZD7762 radiosensitized breast cancer cells with significant effects on p53 mutant cells. (C) Western blot analysis for Chk1 in T47D and MCF7 cells exposed to radiation and/ or AZD7762 in vitro.

A

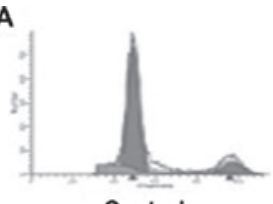

Control

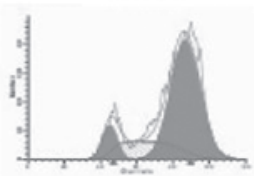

$8 \mathrm{~Gy}$

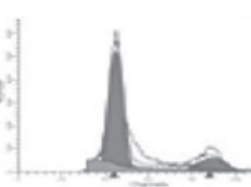

AZD7762

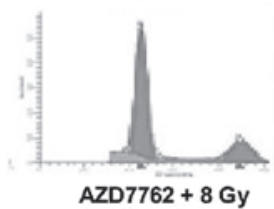

B

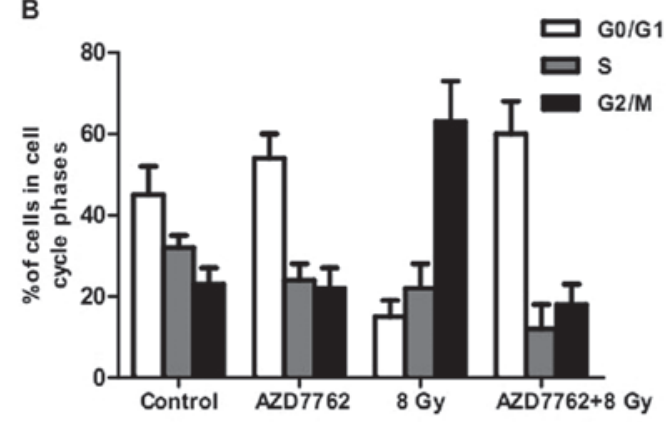

Figure 2. Cell cycle analysis of T47D cells exposed to radiation and/or AZD7762 in vitro. (A) Representative images of cell cycle analysis in the control, AZD7762, radiation and AZD7762 plus radiation groups. T47D cells were treated with AZD7762 (100 nmol/1) $1 \mathrm{~h}$ prior to, and left on for $12 \mathrm{~h}$ after radiation (8 Gy). The cells were then harvested for cell cycle analysis. (B) The bar graph demonstrates that radiation induced G2/M accumulation in breast cancer cells, and the addition of AZD7762 to radiation resulted in the abrogation of the radiation-induced G2/M arrest. Data are representative of 3 independent experiments.

\section{Results}

AZD7762 radiosensitizes breast cancer cells through inhibition of Chkl. The possible radiosensitizing effect of AZD7762 on the p53 mutant T47D and wild-type p53 MCF7 breast cancer cells was examined, using a clonogenic assay. A clearly enhanced radiosensitization of AZD7762 was observed in the p53 mutant T47D cells but not in the wild-type p53 MCF7 cells (Fig. 1A and B). In the p53 mutant T47D cells, the cytotoxicity produced by AZD7762 in combination with radiation was significantly greater than that caused by the radiation alone (colony counts relative to control: AZD7762 plus radiation group vs. AZD7762 and radiation groups, 25.14 $\pm 10.13 \%$ vs. $96.02 \pm 5.41 \%$ and $60.20 \pm 12.02 \%, \mathrm{p}<0.05)$. In the wild-type p53 MCF7 cells, although there was a trend for AZD7762 to sensitize cells to radiation, this difference did not reach a statistical significance (colony counts relative to control: AZD7762 plus radiation group vs. AZD7762 and radiation groups, $39.43 \pm 7.04 \%$ vs. $96.58 \pm 6.13 \%$ and $45.84 \pm 8.13 \%$, $\mathrm{p}>0.05)$. We also aimed to address whether AZD7762 inhibits Chk1 in the two cell lines. Thus, we analyzed Chk1 signaling using western blotting and found that AZD7762 abrogated the radiation-induced Chk1 activation (Fig. 1C). Taken together, these results indicate that AZD7762 radiosensitizes breast cancer cells through the inhibition of Chk1 in a p53-dependent manner.

AZD7762 abrogates radiation-induced G2/M cell cycle arrest. To explore the molecular mechanisms by which AZD7762 radiosensitizes breast cancer cells, we used flow cytometry to define the cell cycle phase distribution of breast cancer cells after radiation. Since the most obvious effects of AZD7762 on the cell cycle phase distribution have been proven to occur at $12 \mathrm{~h}$ after DNA damage, AZD7762 was added to p53 mutant T47D cells $1 \mathrm{~h}$ prior to, and was left on for $12 \mathrm{~h}$ after radiation. Subsequently, the cells were harvested for cell cycle analysis. As shown in Fig. 2A and B, radiation-induced G2/M accumulation in breast cancer cells, and adding AZD7762 to radiation resulted in the abrogation of the radiation-induced $\mathrm{G} 2 / \mathrm{M}$ arrest (percentage of cells in the G2/M phases: AZD7762 plus radiation group vs. control, AZD7762 and radiation groups, $18.37 \pm 5.01 \%$ vs. $23.37 \pm 4.01,22.32 \pm 5.32]$ and $63.41 \pm 10.19 \%$, 
A

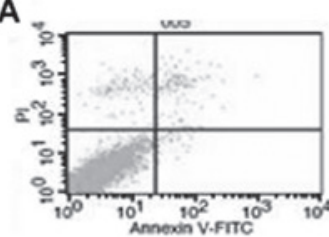

Control

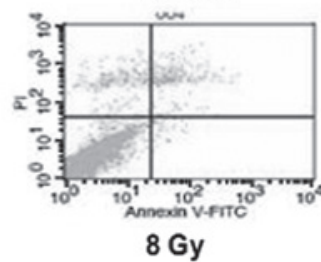

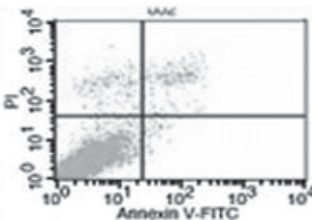

AZD7762

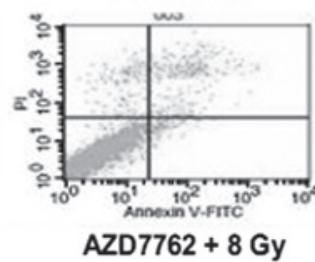

B

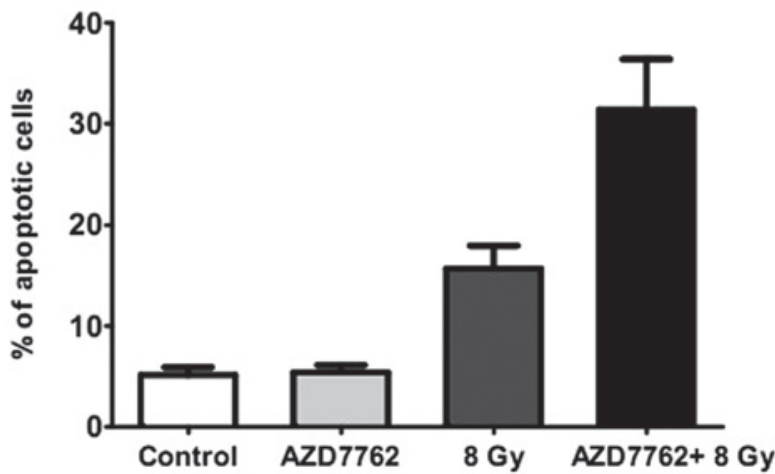

Figure 3. AZD7762 promotes radiation-induced apoptosis. (A) Representative images of cell apoptosis analysis of cells in the control, AZD7762, radiation and AZD7762 plus radiation groups. T47D cells were treated with AZD7762 (100 nmol/1) $1 \mathrm{~h}$ prior to, and left on for $24 \mathrm{~h}$ after radiation (8 Gy). Subsequently, cells were harvested for apoptosis. (B) The bar graph demonstrates that AZD7762 promoted radiation-induced apoptosis in T47D cells. Data are representative of 3 independent experiments.

A

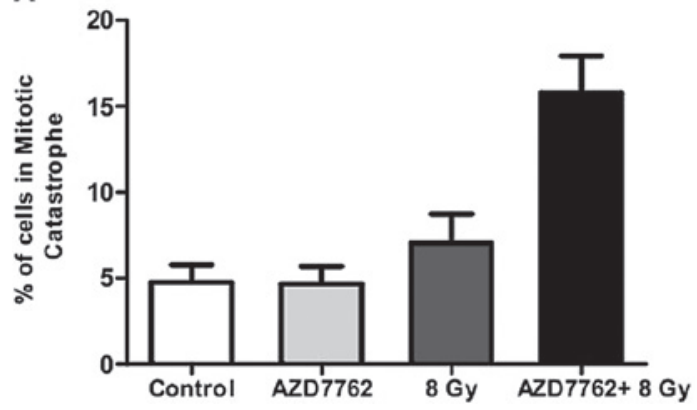

B

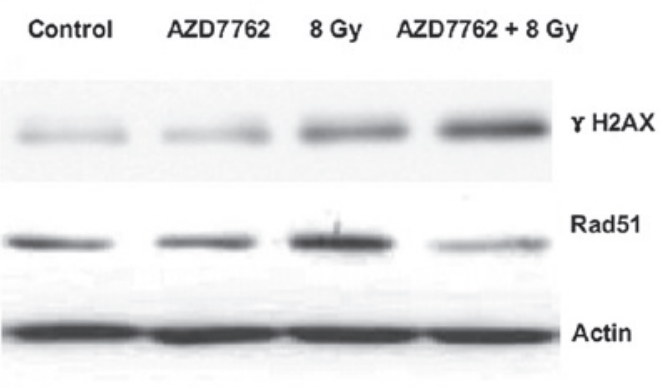

Figure 4. (A) AZD7762 promotes radiation-induced mitotic catastrophe. The mitotic catastrophe analysis demonstrates that AZD7762 promotes radiationinduced mitotic catastrophe in T47D cells. T47D cells were treated with AZD7762 (100 nmol/l) $1 \mathrm{~h}$ prior to, and left on for $24 \mathrm{~h}$ after radiation (8 Gy). Cells were fixed and stained for mitotic catastrophe. Nuclear fragmentation (defined as the presence $\geq 2$ distinct lobes within a single cell) was evaluated in 200 cells per treatment experiment. Data are representative of 3 independent experiments. (B) Western blot analysis for $\gamma \mathrm{H} 2 \mathrm{AX}$ and Rad51 in T47D cells exposed to radiation and/or AZD7762 in vitro.

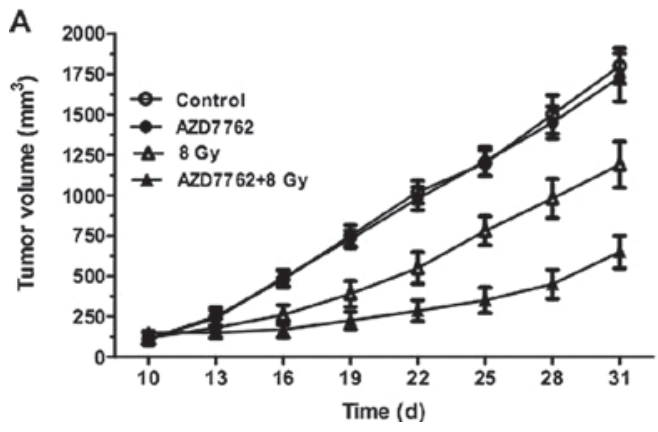

B

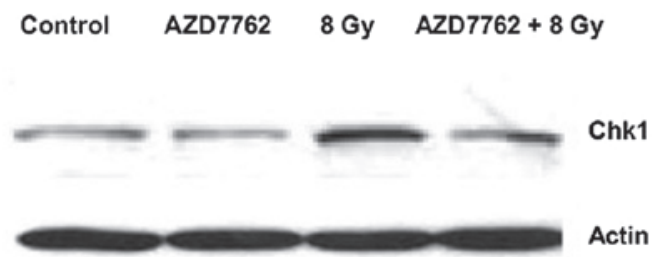

Figure 5. (A) AZD7762 promotes radiation-induced tumor growth delay. Mice were inoculated subcutaneously on the hind flank with T47D cells (1x10 ${ }^{5}$ ). Mice were radiated once a day for 4 days with a dose of 2 Gy per fraction (total 8 Gy). During the radiation treatment, mice were injected with PBS or AZD7762 $(25 \mathrm{mg} / \mathrm{kg}$, i.p.) twice a day for 4 days. Tumor size was assessed by caliper measurements twice a week. Volumes were calculated using the formula $(\mathrm{LxWxW}) / 2$. The data demonstrate that AZD7762 promotes radiation-induced tumor growth delay. (B) Western blot analysis showed that AZD7762 inhibited Chk1 expression in T47D cells xenografts in vivo.

$\mathrm{p}<0.05)$. Taken together, these results support the conclusion that AZD7762 abrogates radiation induced G2/M accumulation in breast cancer cells.

AZD7762 facilitates radiation-induced apoptosis. To gain more insights into the molecular mechanisms by which
AZD7762 radiosensitizes breast cancer cells, we also determined whether the radiosensitization of AZD7762 correlates with apoptosis using flow cytometry. As the DNA damage repair occurs within $24 \mathrm{~h}$ after radiation, p53 mutant T47D cells were treated with AZD7762 $1 \mathrm{~h}$ prior to, and left on $24 \mathrm{~h}$ after radiation. The cells were then harvested for the apoptosis 


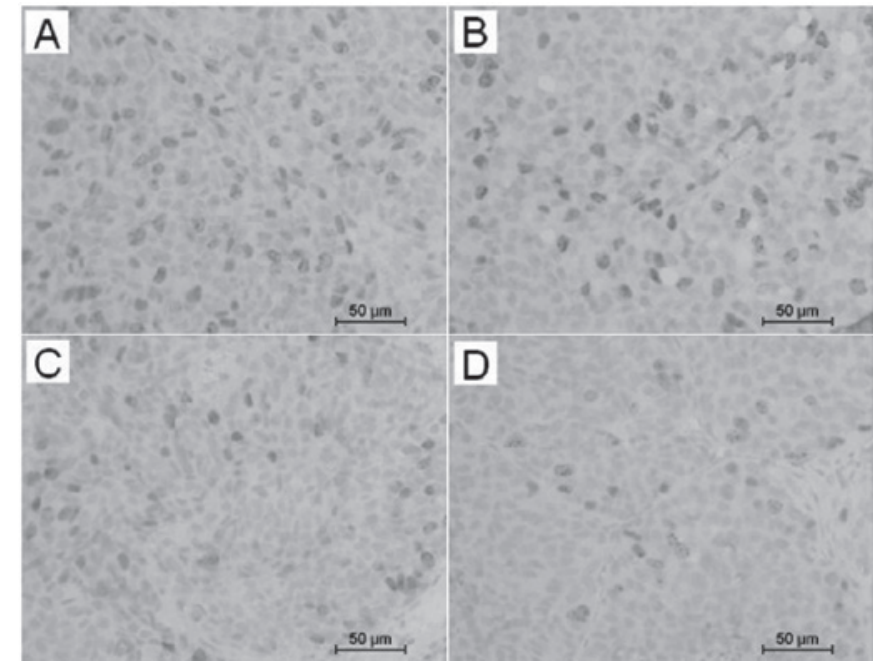

Figure 6. AZD7762 facilitates the radiation-induced proliferative inhibition of tumor growth in vivo. Representive imaging of PCNA immunoreactivity in T47D cell xenografts treated with (A) PBS, (B) AZD7762, (C) radiation or (D) radiation plus AZD7762, respectively. The addition of AZD7762 to the radiation resulted in decreased proliferation in tumor xenografts in vivo.

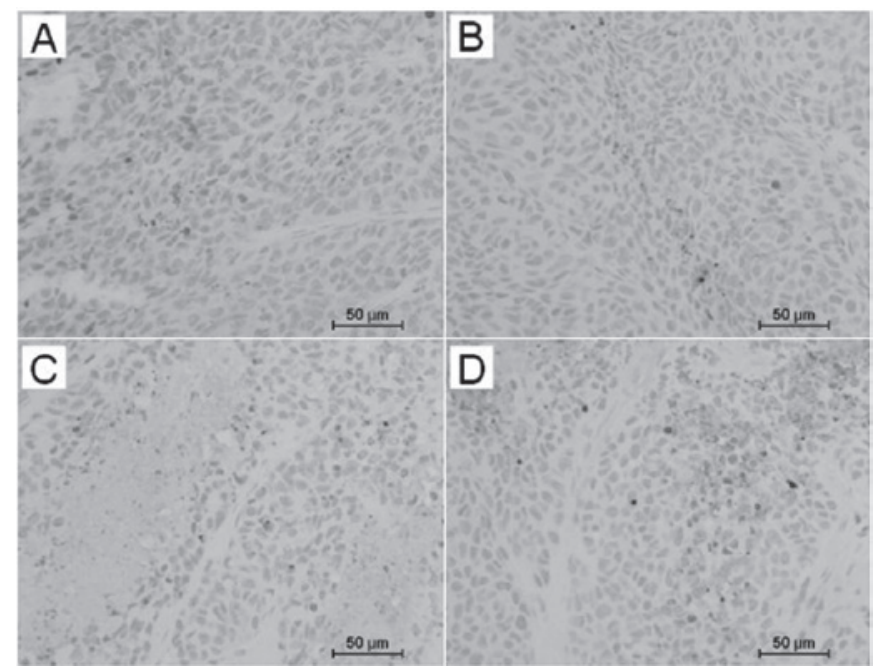

Figure 7. AZD7762 promotes the radiation-induced apoptosis in vivo. Representive imaging of TUNEL staining in T47D cell xenografts treated with (A) PBS, (B) AZD7762, (C) radiation or (D) radiation plus AZD7762, respectively. The addition of AZD7762 to the radiation resulted in increased apoptosis in tumor xenografts in vivo.

analysis. Radiation alone was found to induce the apoptosis of breast cancer cells. As shown in Fig. 3A and B, combining AZD7762 with radiation increased the apoptosis rate significantly beyond the rate observed subsequent to radiation alone (apoptosis percentage: AZD7762 plus radiation group vs. control, AZD7762 and radiation groups, $31.21 \pm 5.34 \%$ vs. $5.16 \pm 1.21 \%, 5.43 \pm 0.96 \%$ and $16.72 \pm 3.12 \%, \mathrm{p}<0.05)$. The results have demonstrate that AZD7762 facilitates radiationinduced apoptosis.

AZD7762 promotes radiation-induced mitotic catastrophe and inhibits the repair of radiation-induced DNA double strand breaks (DSBs). Previous clonogenic assay results demonstrated the enhanced radiation cytotox- icity of AZD7762, and that radiation-induced cell death mainly comprises two major modes: apoptosis and mitotic catastrophe (16-18). Consequently, we hypothesized that, besides apoptosis, AZD7762 radiosensitizes T47D cells by promoting mitotic catastrophe. To confirm this, T47D cells were treated with AZD7762 $1 \mathrm{~h}$ prior to, and was left on $24 \mathrm{~h}$ after radiation. The possible AZD7762 enhancing effect on mitotic catastrophe, which was defined as the presence of $\geq 2$ nuclear lobes within a single cell was measured. As shown in Fig. 4A, we found that AZD7762 significantly promoted radiation-induced mitotic catastrophe in breast cancer cells (AZD7762 plus radiation group vs. control, AZD7762 and radiation groups, $15.53 \pm 4.26 \%$ vs. $4.83 \pm 1.21,4.73 \pm 1.03$ and $7.31 \pm 1.98, \mathrm{p}<0.05)$. Besides mitotic catastrophe, we assumed that AZD7762 was likely to inhibit the radiation-induced DNA DSBs repair. As $\gamma \mathrm{H} 2 \mathrm{AX}$ protein expression is correlated with DSBs, and RAD51 is a protein marker associated with DNA damage repair, the $\gamma \mathrm{H} 2 \mathrm{AX}$ and RAD51 protein expressions were examined by western blotting. AZD7762 plus radiation treatment resulted in an increased radiationinduced $\gamma \mathrm{H} 2 \mathrm{AX}$ expression and a decreased RAD51 protein expression (Fig. 4B). These results indicate that AZD7762 inhibits the repair of radiation-induced DSBs.

AZD7762 sensitizes breast tumor xenografts to radiation. Having established that AZD7762 radiosensitizes breast cancer cells in vitro, and to translate our above in vitro findings into an in vivo model, we evaluated the radiosensitization efficacy of AZD7762 in breast cancer cell tumor xenografts in athymic nude mice. Results showed that AZD7762 significantly delayed the tumor growth in nude mice receiving radiation and, compared to radiation alone, adding AZD7762 to radiation significantly prolonged the time required for tumor volume doubling (Fig. 5A). Additionally, to characterize the molecular mechanisms by which AZD7762 radiosensitizes breast cancer tumor xenografts in vivo, Chk1 signaling was analyzed in tumors receiving AZD7762 plus radiation treatment for 4 days. Consistent with our in vitro findings, Chk1 was inhibited by AZD7762 in the presence of radiation (Fig. 5B). Moreover, as shown in Figs. 6 and 7, adding AZD7762 to radiation resulted in an apoptosis-induced increase and a decreased proliferation evidenced by the TUNEL and PCNA staining in tumor xenografts in vivo (AZD7762 plus radiation xenografts vs. control, AZD7762 and radiation ones, PCNA: $5.70 \pm 1.20$ vs. 20.21 $\pm 5.24,18.2 \pm 6.12$ and $9.32 \pm 3.61 \%$, TUNEL staining: $13.43 \pm 4.26$ vs. $4.43 \pm 2.65,5.31 \pm 1.73$ and $8.21 \pm 2.74 \%, \mathrm{p}<0.05)$. Taken together, these data suggest that AZD7762 sensitizes breast cancer cells to radiation in vivo.

\section{Discussion}

In this study, we found that exposure to AZD7762 may radiosensitize breast cancer cells in vitro and in vivo. These effects may be related to inhibition of the G2/M cell cycle arrest and DNA repair. The inhibition of these two processes results in an increased radiation-induced apoptosis and mitotic catastrophe. Moreover, p53 mutants can predispose breast cancer cells/xenografts to radiation-induced cell death. Our study suggests that Chk1 inhibitor AZD7762 potentially serves as a radiosensitizer in breast cancer tumor with p53 mutations. 
Being a checkpoint inhibitor, Chk1 inhibitor AZD7762 has been proven to enhance the toxicity of various DNA damages (19-22). Our results demonstrate that adding AZD7762 to radiation resulted in profound radiosensitization in the breast tumor cells/xenografts. These data further support the clinical potential of AZD7762 as a radiosensitizing agent in breast cancer therapy. In this study, Chk1 inhibitor AZD7762 significantly radiosensitized breast cancer cells/xenografts with p53 mutants, but not those with wild-type p53 activity. These results are consistent with those of previous studies demonstrating that tumors with p53 mutants may be hyperdependent on the G2 damage-induced checkpoint activity mediated by the Chk1 activity (23-26). These results provide evidence that inhibition of the G2 checkpoint by pharmacological AZD7762 may sensitize p53 mutant breast cancer cells to DNA-damaging agents.

In the present study, the enhanced radiation-induced cell eradication effect of AZD7762 was observed both in a clonogenic and a CCK8 assay (data not shown). It is well-known that cell death induced by radiation comprises two major modes: apoptosis and mitotic catastrophe $(17,27,28)$. We found that the additive radiation-induced apoptosis in response to AZD7762 in this study was not sufficient to account for the enhanced radiation-induced cell-eradicating effect of AZD7762. Therefore, we hypothesized that mitotic catastrophe might also be correlated with the AZD7762 radiosensitization reaction. Thus, we delineated this issue and found a significantly higher number of cells with mitotic catastrophe in the radiation plus AZD7762 group. These results, to some extent, support the hypothesis that mitotic catastrophe is correlated with the enhanced cell eradication induced by adding AZD7762 to radiation.

Inhibition of the G2/M cell cycle checkpoint and the radiation-induced DNA DSBs repair has, in general, been linked to the underlying mechanisms of many radiosensitizers (29-31). In an attempt to reveal the precise mechanisms by which AZD7762 radiosensitizes mammary tumor cells, we also evaluated the cell cycle modulation and DSBs repair activity $(\gamma \mathrm{H} 2 \mathrm{AX}$ protein expression) in the AZD7762 radiosensitization process. As anticipated, adding AZD7762 to radiation significantly abrogated the G2/M cell cycle arrest, increased $\gamma \mathrm{H} 2 \mathrm{AX}$ and decreased the RAD51 protein expression levels in breast cancer cells. These results indicate that the inhibition of the G2/M cell cycle arrest and the radiation-induced DNA DSBs repair may be correlated with the mechanisms underlying the AZD7762 radiosensitization.

There are some inherent limitations in our study. A number of Chk 1 inhibitors, including AZD7762, have been demonstrated to have the undesirable properties of avid binding to human serum proteins and inhibition of other non-checkpoint kinases $(32,33)$. However, along with the thorough ongoing research on Chk1 inhibitor therapy, we believe that the new schedule and analogues of AZD7762 are likely to overcome these limitations in the future. In conclusion, this study has demonstrated that transient pre-treatment with the Chk1 inhibitor AZD7762 at clinically attainable concentrations may effectively enhance radiation-induced mortality in breast cancer cells and tumors with p53 mutants. In addition, inhibition of the G2/M cell cycle checkpoint and the radiationinduced DNA DSBs repair may be the underlying mechanisms, which require further investigation.

\section{References}

1. Truong PT, Olivotto IA, Whelan TJ and Levine M: Clinical practice guidelines for the care and treatment of breast cancer: 16. Locoregional post-mastectomy radiotherapy. CMAJ 170: 1263-1273, 2004.

2. Bartelink H, Horiot JC, Poortmans P, et al: Recurrence rates after treatment of breast cancer with standard radiotherapy with or without additional radiation. N Engl J Med 345: 1378-1387, 2001.

3. Liu Q, Guntuku S, Cui XS, et al: Chk1 is an essential kinase that is regulated by Atr and required for the $\mathrm{G}(2) / \mathrm{M}$ DNA damage checkpoint. Genes Dev 14: 1448-1459, 2000.

4. Kastan MB and Bartek J: Cell-cycle checkpoints and cancer. Nature 432: 316-323, 2004.

5. Gao Q, Huang X, Tang D, et al: Influence of chk1 and plk1 silencing on radiation- or cisplatin-induced cytotoxicity in human malignant cells. Apoptosis 11: 1789-1800, 2006.

6. Bartek J and Lukas J: Chk1 and Chk2 kinases in checkpoint control and cancer. Cancer Cell 3: 421-429, 2003.

7. Koniaras K, Cuddihy AR, Christopoulos H, Hogg A and O'Connell MJ: Inhibition of Chk1-dependent G2 DNA damage checkpoint radiosensitizes p53 mutant human cells. Oncogene 20: 7453-7463, 2001

8. Syljuasen RG, Sorensen CS, Hansen LT, et al: Inhibition of human Chk1 causes increased initiation of DNA replication, phosphorylation of ATR targets, and DNA breakage. Mol Cell Biol 25: 3553-3562, 2005.

9. Pan Y, Ren KH, He HW and Shao RG: Knockdown of Chk1 sensitizes human colon carcinoma HCT116 cells in a p53-dependent manner to lidamycin through abrogation of a G2/M checkpoint and induction of apoptosis. Cancer Biol Ther 8: 1559-1566, 2009.

10. Chen Z, Xiao Z, Gu WZ, et al: Selective Chk1 inhibitors differentially sensitize p53-deficient cancer cells to cancer therapeutics. Int J Cancer 119: 2784-2794, 2006.

11. Zabludoff SD, Deng C, Grondine MR, et al: AZD7762, a novel checkpoint kinase inhibitor, drives checkpoint abrogation and potentiates DNA-targeted therapies. Mol Cancer Ther 7: 2955-2966, 2008.

12. Mitchell JB, Choudhuri R, Fabre K, et al: In vitro and in vivo radiation sensitization of human tumor cells by a novel checkpoint kinase inhibitor, AZD7762. Clin Cancer Res 16: 2076-2084, 2010.

13. Bucher N and Britten CD: G2 checkpoint abrogation and checkpoint kinase-1 targeting in the treatment of cancer. Br J Cancer 98: 523-528, 2008.

14. Morgan MA, Parsels LA, Zhao L, et al: Mechanism of radiosensitization by the Chk1/2 inhibitor AZD7762 involves abrogation of the G2 checkpoint and inhibition of homologous recombinational DNA repair. Cancer Res 70: 4972-4981, 2010.

15. Maity A, McKenna WG and Muschel RJ: The molecular basis for cell cycle delays following ionizing radiation: a review. Radiother Oncol 31: 1-13, 1994.

16. Held KD: Radiation-induced apoptosis and its relationship to loss of clonogenic survival. Apoptosis 2: 265-282, 1997.

17. Vakifahmetoglu H, Olsson M and Zhivotovsky B: Death through a tragedy: mitotic catastrophe. Cell Death Differ 15: 1153-1162, 2008.

18. Brown JM and Attardi LD: The role of apoptosis in cancer development and treatment response. Nat Rev Cancer 5: 231-237, 2005.

19. Aris SM and Pommier Y: Potentiation of the novel topoisomerase I inhibitor indenoisoquinoline LMP-400 by the cell checkpoint and Chk1-Chk2 inhibitor AZD7762. Cancer Res 72: 979-989, 2012.

20. Vance S, Liu E, Zhao L, et al: Selective radiosensitization of p53 mutant pancreatic cancer cells by combined inhibition of Chk1 and PARP1. Cell Cycle 10: 4321-4329, 2011.

21. Bartucci M, Svensson S, Romania P, et al: Therapeutic targeting of Chk1 in NSCLC stem cells during chemotherapy. Cell Death Differ 19:368-378, 2011.

22. Yang H, Yoon SJ, Jin J, et al: Inhibition of checkpoint kinase 1 sensitizes lung cancer brain metastases to radiotherapy. Biochem Biophys Res Commun 406: 53-58, 2011.

23. Wilsker D, Chung JH and Bunz F: Chk1 suppresses bypass of mitosis and tetraploidization in p53-deficient cancer cells. Cell Cycle 11: 1564-1572, 2012.

24. Origanti S, Cai SR, Munir AZ, White LS and Piwnica-Worms H: Synthetic lethality of Chk1 inhibition combined with p53 and/or p21 loss during a DNA damage response in normal and tumor cells. Oncogene: Mar. 19, 2012 (E-pub ahead of print). 
25. Tse AN, Sheikh TN, Alan H, Chou TC and Schwartz GK: 90-kDa heat shock protein inhibition abrogates the topoisomerase I poison-induced G2/M checkpoint in p53-null tumor cells by depleting Chk1 and Wee1. Mol Pharmacol 75: 124-133, 2009.

26. Jia XZ, Yang SY, Zhou J, et al: Inhibition of CHK1 kinase by Go6976 converts 8-chloro-adenosine-induced G2/M arrest into $\mathrm{S}$ arrest in human myelocytic leukemia K562 cells. Biochem Pharmacol 77: 770-780, 2009.

27. Eriksson D and Stigbrand T: Radiation-induced cell death mechanisms. Tumour Biol 31: 363-372, 2010.

28. Roninson IB, Broude EV and Chang BD: If not apoptosis, then what? Treatment-induced senescence and mitotic catastrophe in tumor cells. Drug Resist Updat 4: 303-313, 2001.

29. Kesari S, Advani SJ, Lawson JD, et al: DNA damage response and repair: insights into strategies for radiation sensitization of gliomas. Future Oncol 7: 1335-1346, 2011.
30. McNeely S, Conti C, Sheikh T, et al: Chk1 inhibition after replicative stress activates a double strand break response mediated by ATM and DNA-dependent protein kinase. Cell Cycle 9: 995-1004, 2010.

31. O'Connor MJ, Martin NM and Smith GC: Targeted cancer therapies based on the inhibition of DNA strand break repair. Oncogene 26: 7816-7824, 2007.

32. Tao ZF and Lin NH: Chk1 inhibitors for novel cancer treatment. Anticancer Agents Med Chem 6: 377-388, 2006.

33. Ashwell S, Janetka JW and Zabludoff S: Keeping checkpoint kinases in line: new selective inhibitors in clinical trials. Expert Opin Investig Drugs 17: 1331-1340, 2008. 